\title{
AN EMBEDDED SYSTEM FOR SMALL-SCALED AUTONOMOUS VEHICLES
}

\author{
David Vissière and Nicolas Petit \\ Délégation Générale pour l'Armement, France \\ École Nationale Supérieure des Mines de Paris, France \\ david.vissiere@dga.defense.gouv.fr,nicolas.petit@cas.ensmp.fr
}

Keywords: Embedded systems, autonomous vehicles, UAVs.

\begin{abstract}
We consider the problem of designing a modular real-time embedded system for control applications with unmanned vehicles. We propose a simple and low-cost solution. Its computational power can be easily improved, depending on application requirements. To illustrate its performance, we report the implementation of a $75 \mathrm{~Hz}$ Extended Kalman Filter used for state estimation on a small-scaled helicopter.
\end{abstract}

\section{INTRODUCTION}

In this paper, we present some of our research effort in a current program aiming at proposing control strategies and a control architecture for a group of heterogeneous autonomous vehicles.

To conduct this research, we designed a versatile and simple real time embedded system, which can be easily used as real time guidance and navigation system on various platforms. Our work focuses on heterogenous vehicles, including small-scaled (typically less than $2 \mathrm{~m}$ wide) Vertical Take-Off and Landing aerial vehicles (VTOL as in (Castillo et al., 2005)) or fixed wing aircraft, and ground vehicles with tank like dynamics (as in (Morin and Samson, 2006; Vissière et al., 2007)). In the future, these vehicles will be asked to act cooperatively on the battlefield as pictured in Figure 1 (see also (Kaminer et al., 2004; Olfati-Saber, 2006) for other scenarios).

In practice, the aerial vehicles represent the most challenging applications in terms of navigation and guidance. The main reason for this, is that these vehicles can not easily go into any safe mode, as opposed to the ground vehicles which are, in comparison, slower and simpler. While it was proven that, with lowered performance expectations, it is possible to stabilize a fixed-wing Unmanned Air Vehicles (UAV) by directly closing the loop with signals from well-chosen sensors (e.g. in (Lee et al., 2003), the authors propose a solution to automatically control a fixed-wing UAV using only a single-antenna GPS receiver), it is considered by the vast majority of the $\mathrm{UAV}$ community that navigation systems require data fusion (Cheng et al., 2006). In facts, each sensor technology has its own flaws (among which are drift, noises, and possibly low resolution or low update frequency). Yet, large factors of accuracy can be gained by reconciliating their data.

Example of on-board data fusion applications are ubiquitous among autonomous vehicle control experiments. Reconciliating GPS and Inertial Measurement Unit (IMU) measurements is a classic case-study. In (Xiaokui and Jianping, 2002), results of data fusion from a BeeLine GPS receiver from Novatel ${ }^{\mathrm{TM}}$ and a miniaturized IMU are presented. In (Cremean et al., 2005), high-speed data fusion systems have been developed in view of the DARPA Grand Challenge.

In this later experiment, several technological breakthroughs are presented using a high-end and powerful computer architecture. Software components communicate in a machine-independent fashion through a module management system.

Our experiments can not use such a high end setup, because the typical payload of our aerial vehicles does not exceed $5 \mathrm{~kg}$.

Much smaller and lower-weighting systems can be considered though. In (Jung and Tsiotras, 2007), an embedded system is proposed which does not incorporate any powerful calculation board. A simple Rabbit Semiconductor RCM-3400 ${ }^{\mathrm{TM}}$ microcontroller is used to perform complementary filtering data fusion using a limited computational power. In the same spirit, in (Jung et al., 2005), a low-cost testbed for UAVs is presented. It is reported that the main advantage of designing such an autopilot from 


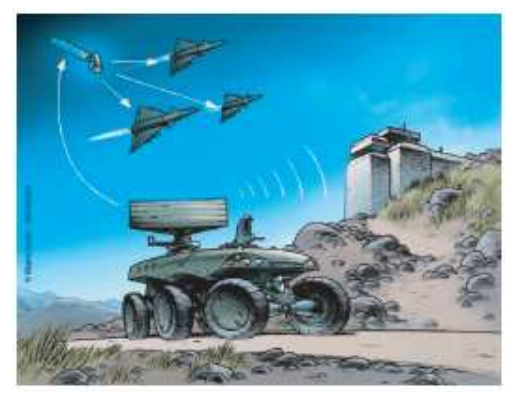

Figure 1: Cooperative autonomous vehicles in a future battlefield.

scratch is that, by contrast to commercially available products (Cloud Cap Technologies, 2004; Micro Pilot, 2004), it provides full access to the internal control structures. We totally agree with this point of view.

In this paper, we present a solution lying in the middle of the two previously mentioned categories. Our system uses two processors. One processor is used to gather data from the sensors and to control the actuators. The other processor is used to perform the data fusion calculations (and possibly the control algorithms). The advantages of this structure are as follows: $i$ ) task scheduling is easily programmed, because only one of the two processors is in charge of handling the numerous devices and $\mathrm{I} / \mathrm{O}$; ii) the computations are performed as one single thread on a dedicated board (PC type); iii) depending on the computational requirements, the computation board can be easily upgraded without requiring any software changes or rising any concern about task scheduling; $i v$ ) finally, the overall system is quite low-cost, since it relies on off-the-shelf components and can be easily maintained.

The paper is organized as follows. In Section 2, we present our system architecture. We detail our hardware components and comment on their choices. In Section 3, we present as a test-case the embedding of our system into a small-scaled helicopter. Numerous details of implementation are provided. Finally, we conclude and give directions of future work.

\section{SYSTEM ARCHITECTURE}

Our primary goal was to develop an embedded system to test algorithms of various complexity on-board various small-scaled platforms. Early in the design process, one first constraint which appeared to us was the payload limitations of the considered flying machines. This lead us to focus on designing a low weight embedded system.
A second issue that was also raised early in the design stage was that the real-time requirements of a control system for such small UAVs are very strong. This is mostly due to the short time horizons instabilities. Yet, in the context of embedded systems, realtime scheduling of a number of sensing and computation tasks is known to be a difficult problem. More precisely, as exposed in (Caccamo et al., 2005), the problem of determining the feasibility of a periodic sequence of prioritized tasks is often (NP)-hard. Sufficient, but not necessary tests are pessimistic. Popular strategies such as the Rate-Monotonic policy (see again (Caccamo et al., 2005)), which consists of putting the highest priority on the shortest task can be proven to be unfeasible is the CPU load is too large ${ }^{1}$. While being troublesome on ground vehicles, such infeasibilities (and the induced inconsistencies in the embedded calculations) would represent a cause of potential major failure for our aerial platforms.

Keeping these two considerations in mind, we decided to develop a robust two-processors embedded system, running two distinct softwares and communicating through a simple two-ways protocol. The system specifications are as follows.

1. It performs the sensing and calculation tasks separately.

2. It is fast enough to run a typical 15 to 30 dimensional states EKF algorithm with a low latency (to eventually produce satisfactory closed-loop results).

3. Itis easy to upgrade.

4. It is versatile enough to handle various type of sensors and communication protocols.

As exposed in Figure 2 and Figure 3, this (modular) embedded system is composed of a microcontroller, which is in charge of gathering information from all the sensors, and a calculation board. These two elements are connected by a serial interface. The micro-controller also has a downlink to a ground station. We now present the details of the hardware components of our system.

\subsection{Sensors}

Considering both ground and aerial vehicles control applications, we listed a series of useful sensors that needed to be incorporated into our embedded system. Among these are: an IMU, a GPS receiver, a pressure sensor, an anemometer, magnetometers, and various switches. Other possibilities include odometers,

\footnotetext{
${ }^{1}$ the upper limit on admissible load is $69 \%$, approximately.
} 
LADARs (as used in (Cremean et al., 2005)), and sonars (as used in (Vissière et al., 2007)), or cameras (as used in (Hamel and Mahony, 2007)). In the context of our study, we only considered low-cost sensors. We now detail these. In each case, we specify the weight (in $g$ ), the cost (in USD), the dimensions (in $\mathrm{mm}$ ), the update rate ( $\mathrm{f}$ in $\mathrm{Hz}$ ), and the protocol of communication (Comm.).

- Inertial Measurement Unit (IMU). Our IMU is a 3DM-GX1 from Microstrain ${ }^{\mathrm{TM}}$. It contains three angular rate gyroscopes, three orthogonal single-axis magnetometers, and three single-axis accelerometers, along with 16 bits A/D converters and a micro-controller. This IMU can deliver different messages, ranging from raw-data, to reconciliated measurements. In our setup, we ask the IMU to deliver only calibrated sensors data at a $75 \mathrm{~Hz}$ rate.

\begin{tabular}{|c|c|c|c|c|}
\hline Weight & Cost & Size & f & Comm. \\
\hline 30 & 1450 & $39,54,18$ & 75 & RS232 \\
\hline
\end{tabular}

- Global Positioning System (GPS). Our GPS is a TIM-LS from $\mu$ blox ${ }^{\text {TM }}$. Through a proprietary binary protocol, it provides position and velocity information at a $4 \mathrm{~Hz}$ rate. Position error is $2.5 \mathrm{~m}$ (Circular Error Probability CEP) and velocity error is $2 \mathrm{~m} \mathrm{CEP}$.

\begin{tabular}{|c|c|c|c|c|}
\hline Weight & Cost & Size & f & Comm. \\
\hline 23 & 100 & $32,47,9.5$ & 4 & RS232 \\
\hline
\end{tabular}

The GPS receiver is not very tolerant against power supply voltage ripples. These can be kept below the $50 \mathrm{mV}$ requirements thanks to a dedicated power supply regulator from TRACO ${ }^{\mathrm{TM}}$.

- Barometer. Our barometer is the MS-5534 from Intersema $^{\mathrm{TM}}$. Using a SPI-type protocol, it gives calibrated digital pressure and temperature information. This device requires a $3 \mathrm{~V}$ power supply which is obtained through a fast response diode from the main $5 \mathrm{~V}$ power supply of the microcontroller.

\begin{tabular}{|c|c|c|c|c|}
\hline Weight & Cost & Size & f & Comm. \\
\hline 2 & 14 & $5,4,2$ & 20 & SPI \\
\hline
\end{tabular}

- Anemometer. A PXLA sensor from ASensTec ${ }^{\mathrm{TM}}$ delivers a differential pressure analog signal, which can be read through a 10 bit A/D converter.

\begin{tabular}{|c|c|c|c|c|}
\hline Weight & Cost & Size & f & Comm. \\
\hline 10 & 25 & $11,8,12$ & 75 & Analog \\
\hline
\end{tabular}

- Magnetometer. We use a HMR2300 three axis magnetometer from Honeywell ${ }^{\mathrm{TM}}$. Its range is \pm 2 gauss and it has a $70 \mu$ gauss resolution.

\begin{tabular}{|c|c|c|c|c|}
\hline Weight & Cost & Size & f & Comm. \\
\hline 28 & 230 & $75,30,20$ & 154 & RS232 \\
\hline
\end{tabular}

- Take-off and Landing Detector. Being able to detect take-off and landing instants is necessary to properly initialize data fusion algorithms. In details, detection of the corresponding switches in the dynamics defines when the controls actually have an effect on the system. This is not the case when an UAV is on the ground. This detection is performed with on-off switches which can be located, e.g., on the landing gear. They deliver a logic signal which can be readily interpreted. To prevent electric arcs which might cause trouble to the connected micro-controller, we added a specific interfacing circuit. This switches can also be replaced by active switches which can be used to activate various devices such as digital cameras, or parachutes.

\begin{tabular}{|c|c|c|c|c|}
\hline Weight & Cost & Size & f & Comm. \\
\hline 10 & 6 & $25,10,5$ & 75 & Boolean \\
\hline
\end{tabular}

Our system is data-driven by the IMU. The main reason behind this choice is that the IMU is considered as a critical sensor.

\subsection{MPC555 Micro-controller}

The micro-controller which serves as an interface for the sensors and actuators is a MPC555 Power $\mathrm{PC}$ from Motorola ${ }^{\mathrm{TM}}$. It runs a specific software we developed using the Phytec ${ }^{\mathrm{TM}}$ development kit. The reason for choosing this micro-controller are as follows. This device provides a double precision floating-point unit (64 bit) which is convenient for potential embedded algorithms (even if we do not use this possibility here since all computations are performed on the calculation board), it has a relatively fast $40 \mathrm{MHz}$ clock, it has 32 bit architecture and 448Kbyte of Flash memory and 4 MBytes of RAM. Most importantly, among the family of 32 bits kits, the MPC555 has substantial computational capabilities and a large number of versatile and programmable Input/Output ports. In particular, we make an extensive use of its TPUs (Time Process Units), UARTs, A/D converters, SPIs, MPIOs (Modular I/O system) (see (Motorola, 2000)). Finally, it is small (credit card format) and has a low weight.

\begin{tabular}{|c|c|c|c|c|}
\hline Weight & Cost & Size & f & Comm. \\
\hline 25 & 450 & $72,57,8$ & all & all \\
\hline
\end{tabular}

No operating system is used on the microcontroller. Rather the MPC555 runs a specific interrupts-driven software written in C. Information 


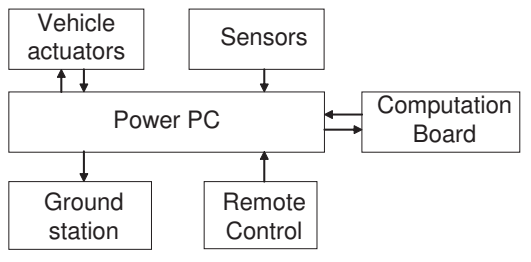

Figure 2: Sensors and computation board connections to the central micro-controller.

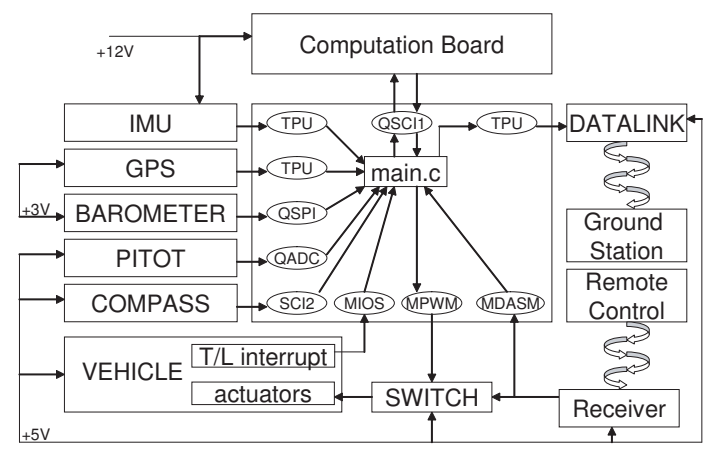

Figure 3: Embedded system internal connections.

from each sensors is transferred using a dedicated interrupt handler routine. Each external source or group of sources has its own interrupt level which avoids potential conflicts. Each data link is associated with a checksum to validate reception.

The data acquisition software running on the micro-controller is event-driven by the IMU messages which periodically sends 31 bytes of data. Once the message of the IMU is received and validated by the micro-controller, others sensors information are either directly read or picked in data buffers which are constantly fed with serial messages from the sensors through hardware interrupts. Information is gathered in a 116 bytes message containing all the onboard measurements. This message is sent to the calculation board through a high-speed serial port. Once the message is received and validated, the calculation board carries out one navigation loop consisting of a prediction equation and an estimation equation of a Kalman filter.

\subsection{PC Computation Board}

The computing board is a PC running the Knoppix 3.8.1 Linux distribution. The PC board was selected among numerous models (mostly mini-ITX, and PC104) based on computational power, energy consumption, toughness, and price. A fan-less board was considered as the most relevant choice, due to the often observed failures of fans in mechanically disturbed environments.
The chosen fan-less calculation board has a standard mini-ITX PC architecture. Its processor is a 1.2Ghz C7-M from VIA ${ }^{\mathrm{TM}}$ designed for embedded applications. It can perform 1500 MIPS and has classic PC Input/Output ports such as a UART serial port (used as main data link with the micro-controller), an ethernet board (not used here), a VGA screen output (which can be used to monitor the system during debugging phases of the software and hardware development), a keyboard, and 4 USB ports (which can be considered for plugging future devices such has controllable cameras).

Experimental preliminary tests have shown us that multi-threading (one thread for message decoding and one thread for the main algorithm) presents two major drawbacks: some data can be lost, and the calculation cycle may end unexpectedly (slightly) late. For this reason, we decided to write our own UART driver using an interrupt handler in the kernel space. Further, we de-activated all hardware interruptions associated to unnecessary devices. As a result, only interrupts from the UART are enabled. Finally, we used a single computation thread.

The operating system is installed on a bootable 1 Gbyte Disk-On-Chip system which prevents all possible mechanical failure associated to hard-drives. This flash memory device is directly connected to the IDE port of the mother-board. The board is powered by a pico-PSU ${ }^{\mathrm{TM}}$ power supply which provides various voltages ranging from $5 \mathrm{~V}$ to $18 \mathrm{~V}$. The computation software are written in $\mathrm{C}$ and can be either be updated directly on the board via a ssh connection, or transferred, in a compiled form, from a remote PC. Custom scripts for compiling and distributing our executable code and configuration files are an efficient way to upgrade the software during development and testing.

\begin{tabular}{|c|c|c|c|c|}
\hline Weight & Cost & Size & f & Comm. \\
\hline 800 & 350 & $170,180,40$ & $1.5 \mathrm{e} 6$ & RS232 \\
\hline
\end{tabular}

\section{FIELD EXPERIMENT}

We now report some field experiment using our embedded system. To perform model identification tasks and design a real-time state observer in view of closed-loop control, we decided to load our embedded system into a small-scaled helicopter. In this section, we expose this work, and give numerous details about solutions to specific interfacing and vibrational issues. 


\subsection{Experimental Setup}

We conducted all the experiments with the help of a human pilot which could, at any time remotely connect the inputs of the actuators of the helicopter to the outputs of our embedded system or directly to the outputs of the radio receiver (and thus take direct control of the helicopter). This is achieved thanks to a "remotely controlled switch board" which we designed. This switch is actuated from our Graupner ${ }^{\mathrm{TM}}$ MC24 remote controller using one of the 12 available radio channels. This system provides a rapid transition between manual and autonomous flight.

During all flights, the pilot could see images from an on-board camera and read measurements from the embedded system received for the down datalink. A convenient Head-Up display system on the ground station was designed for that purpose. Our data link is a MaxStream ${ }^{\mathrm{TM}}$ module operating at $2.4 \mathrm{GHz}$. It provides a RS232 serial data link at 9600 baud sending the information from the embedded system to the ground station.

\begin{tabular}{|c|c|c|c|c|}
\hline Weight & Cost & Size & f & Comm. \\
\hline 250 & 230 & $40,68,9$ & 30 & RS232 \\
\hline
\end{tabular}

\subsection{Small-scaled Helicopter}

We use a Benzin acrobatic helicopter from Vario ${ }^{\mathrm{TM}}$, with Graupner ${ }^{\mathrm{TM}}$ electronics (C4441 servomotors which have high speed and high torque $(8.5 \mathrm{Nm})$, a 16 channels receiver, and a yaw hobby gyroscope). It is a very reliable helicopter platform (we never observed any mechanical issue over mode than 100 flights). Its payload capacity $(4 \mathrm{Kg})$ is large enough for numerous reconnaissance applications. Its rotor is $1.9 \mathrm{~m}$ wide.

\subsection{Interfacing and Vibrational Issues}

Wiring the embedded system to the existing helicopter circuitry was achieved using some specific additional boards and connectors. To measure the pilot's orders in real-time, we used a 6 channels voltage follower circuit. Numerous LEDs were added to check the status of our system.

A central problem observed on-board helicopters is the $25 \mathrm{~Hz}$ vibrations induced by the main rotor blades. These vibrations generate a large amount of noise on the inertial sensors. In practice, these noises totally overwhelm the useful signals. Fortunately, it is possible to solve this issue by using well-chosen noise dampers. On our helicopter, we decided that the micro-controller and the sensors would all be located on a board which would be physically connected to the frame of the helicopter through four

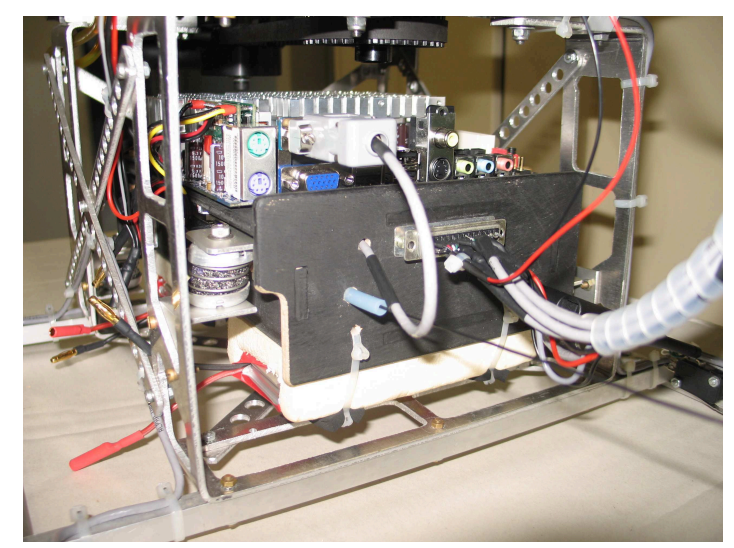

Figure 4: Our embedded system fitted into the (custombuilt) landing gear of a small-scaled Vario Benzin helicopter. Springs and dampers are used to filter out vibrations from the main rotor blades.

spring-damper systems (see Figure 4). Experiments conducted on a vibrating table have shown that it was advantageous to keep the embedded system as compact and as rigid as possible. The total weight of the subsystem is about $600 \mathrm{~g}$. We decided to attach some of the batteries to it to bring the weight close to $1.8 \mathrm{~kg}$. This enabled us to use off-the-shelf dampers yielding appropriate cut-off frequencies. MV801-5CC dampers from Paulstra ${ }^{\mathrm{TM}}$ were chosen for their ability to work with low masses vibrating at low frequencies. With these, we obtained a satisfactory vibration damping, with a cut-off frequency around $9 \mathrm{~Hz}$. This is represented in Figure 5. Further, resonant frequencies due to the engine frequency (around $160 \mathrm{~Hz}$ ), the tail rotor frequency (around $115 \mathrm{~Hz}$ ), and the tail boom were removed using a digital notch filter. The presented solution attenuates high frequency vibration inputs down to negligible levels.

\subsection{Experimental Identification}

Preliminary model identification experiments need to be conducted before state estimation can be performed.

In particular, using our embedded system, we studied the actuators transfer functions and the yaw rate gyroscope.

In some works (see (Mettler, 2003) or (Mettler et al., 2000)), actuators (servomotors) are considered as first order systems with dead band. We identified such transfer functions for various Graupner ${ }^{\mathrm{TM}}$ and Futaba ${ }^{\mathrm{TM}}$ servomotors. Results of various tip-in and tip-out in reference signals were recorded to compute the time constant of the first order model.

On board our helicopter, a hobby gyroscope from 


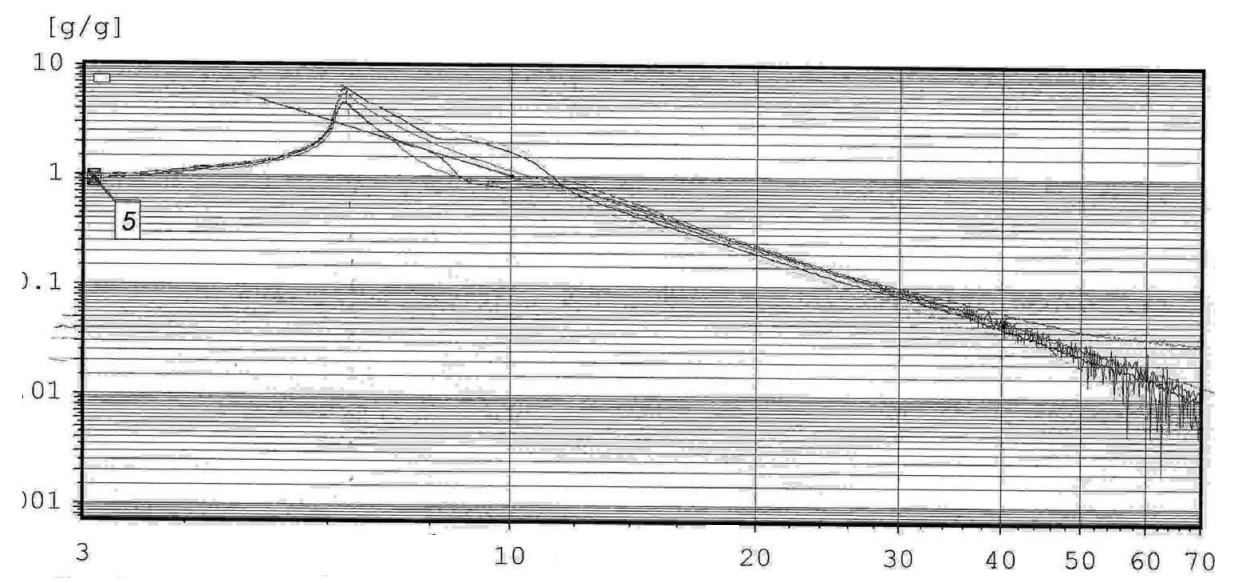

Figure 5: Bode diagram showing the resonance peak and the cut-off frequency of the mechanical structure equiped with the sensors, the micro-controller, and the spring-dampers suspension. The various plots are obtained on varying locations on the vibrating structure and show a good spatial uniformity of the vibration damping.

Graupner $^{\mathrm{TM}}$ is used to help the human pilot keeping the yaw rate as small as possible. Pilot orders are transferred from the $\mathrm{R} / \mathrm{C}$ receiver to the tail actuator through this gyroscope.

To validate simple models of this transfer, we put our IMU under this gyroscope to measure the angular velocity. Simultaneously, we connected the gyroscope and recorded the gyroscope signals sent to the tail actuator. Surprisingly, it was discovered that the gyroscope feedback behaves as a $2 \mathrm{~Hz}$ low-pass filter on the pilot orders, and directly transmits the opposite of the received angular rate, without filtering it. This can be summarized under the form

$$
\delta_{\text {gyro }}=N_{r} r_{m}+\frac{\delta_{\text {pilot }}}{1+\tau_{\text {gyro }} S}
$$

\subsection{State Estimation}

The helicopter is a 6 degrees of freedom mechanical system with high bandwidth dynamic. Reconstructing the full state of this system from low-cost sensors only is quite a challenge. We use an Extended Kalman Filter (EKF, see e.g. (Simon, 2005)) to estimate the state of this system. In practice, the state of our EKF is composed of 23 variables including configuration states (13 variables using quaternions instead of Euler angles), and the aerodynamic and external forces and torques (10 variables including the harmonic expansion of the flapping phenomena as exposed in (Mettler et al., 2001)). We used equally valued tuning parameters for the 3 axis. These are chosen to capture fast dynamics (statistics data are $\sigma_{\text {acceleration }}=8 \mathrm{~m} . \mathrm{s}^{-2}$,

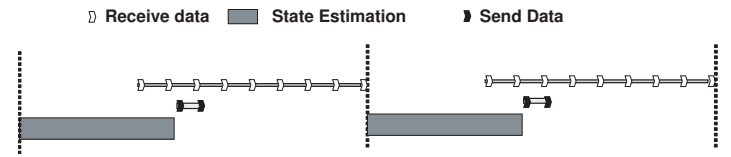

Figure 6: Succession of steps of data transfer and computation.

and $\sigma_{\text {torque }}=4{\mathrm{rad} . \mathrm{s}^{-2}}$, respectively). Classically, discrete-time updates are implemented. As already discussed, updates are synchronized with the $75 \mathrm{~Hz}$ measurements from the IMU.

The succession of tasks performed by the computation board of our embedded system is described in Figure 6. As can be seen, it is necessary, due to a reception time being superior to the available time between two calculation cycles to simultaneously read or send data and perform computations. Some experimental results are presented in Figure 7. Position estimates around hovering are reported. In practice, they appear to be in great accordance with recorded videos.

\section{CONCLUSIONS}

Designing an embedded system which can qualify as a control system for various small-scaled air and ground vehicles is the subject of the research project presented in this paper. The embedded system we propose here has some interesting features. It is simple, low cost and, most of all, easy to upgrade. Its two processors architecture can incorporate various new 


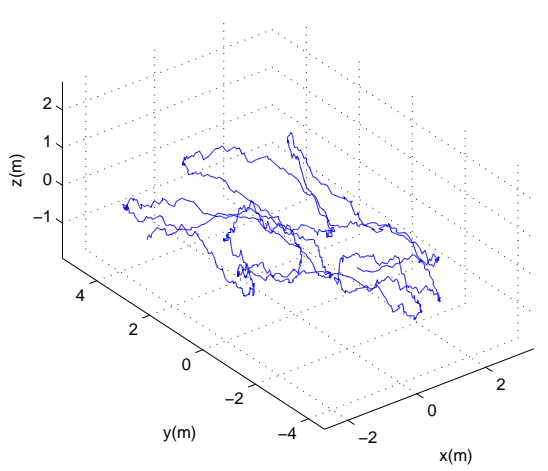

Figure 7: Position estimates during a hovering flight.

processors and sensors.

In our laboratory, this embedded system has been successfully used on a fixed wing aircraft (Rascal 110), a small-scaled helicopter (Vario ${ }^{\mathrm{TM}}$ Benzin trainer), and ground vehicles (Pioneer 4 from Mobile Robots $^{\mathrm{TM}}$ ).

Further developments focus on giving more autonomy, including path planning algorithm, following (Kogan and Murray, 2006; Murray et al., 2003), to respond to high-levels orders from a remote user. We will surely need more computational power, which can be obtained by simply upgrading the computational board. In parallel, we develop a new aerial vehicle for urban area applications. For this last project we consider using other sensors. In particular, for obstacle avoidance and navigation, we will connect LADARS, and ultrasonic sensors to our embedded system.

\section{ACKNOWLEDGEMENTS}

The authors are indebted to the numerous students, technicians and engineers who have been collaborating to the development of the presented technology and have brought their support during the conducted experiments.

\section{REFERENCES}

Caccamo, M., Baker, T., Burns, A., Buttazzo, G., and Sha, L. (2005). Real-time scheduling for embedded systems. In Hristu-Varsakelis, D. and Levine, W. S., editors, Handbook of networked and embedded control systems. Birkhäuser.

Castillo, P., Lozano, R., and Dzul, A. E. (2005). Modelling and control of mini-flying machines. Advances in industrial control. Springer.
Cheng, J., Lu, Y., Thomas, E. R., and Farrell, J. A. (2006) Data fusion via Kalman filter: GPS and INS. In Ge, S. S. and Lewis, F. L., editors, Autonomous mobile robots, Control engineering series. Taylor and Francis.

Cloud Cap Technologies (2004). http:// www.cloudcaptech.com. Hood River, OR, USA.

Cremean, L. B., Foote, T. B., Gillula, J. H., Hines, G. H., Kogan, D., Kriechbaum, K. L., Lamb, J. C., Leibs, J., Lindzey, L., Rasmussen, C. E., Stewart, A. D., Burdick, J. W., and Murray, R. M. (2005). Alice: An information-rich autonomous vehicle for high-speed desert navigation. Journal of Field Robotics, (9).

Hamel, T. and Mahony, R. (2007). Image based visual servo-control for a class of aerial robotic systems. 43(11):1975-1983.

Jung, D., Levy, E. J., Zhou, D., Fink, R., Moshe, J., Earl, A., and Tsiotras, P. (2005). Design and development of a low-cost test-bed for undergraduate education in UAVs. In Proc. of the 44th IEEE Conf. on Decision and Control, and the European Control Conference 2005.

Jung, D. and Tsiotras, P. (2007). Inertial attitude and position reference system development for a small UAV. In AIAA Infotech at Aerospace.

Kaminer, I., Yakimenko, O., Dobrokhodov, V., Lizaraga, M., and Pascoal, A. (2004). Cooperative control of small uavs for naval applications. In Proc. of the $43 \mathrm{rd}$ IEEE Conf. on Decision and Control.

Kogan, D. and Murray, R. M. (2006). Optimization-based navigation for the DARPA Grand Challenge. In Proc. of the 45th IEEE Conf. on Decision and Control.

Lee, S., Lee, T., Park, S., and Kee, C. (2003). Flight test results of UAV automatic control using a single-antenna GPS receiver. In AIAA Guidance, Navigation, and Control Conference and Exhibit.

Mettler, B. (2003). Identification Modeling and Characteristics of Miniature Rotorcraft. Boston : Kluwer Academic Publishers.

Mettler, B., Tischler, M. B., and Kanade, T. (2000). System identification of a model-scale helicopter. Technical Report CMU-RI-TR-00-03, Carnegie Mellon University.

Mettler, B., Tischler, M. B., and Kanade, T. (2001). System identification modeling of a small-scale unmanned helicopter. Journal of the American Helicopter Society.

Micro Pilot (2004). http://www.micropilot.com. Stony Mountain, Canada.

Morin, P. and Samson, C. (2006). Trajectory tracking for nonholonomic vehicles. In Kozlowski, K., editor, Robot Motion Control: Recent Developments. Springer.

Motorola (2000). MPC555 / MPC556 user's manual. User's manual, Motorola.

Murray, R. M., Hauser, J., Jadbabaie, A., Milam, M. B., Petit, N., Dunbar, W. B., and Franz, R. (2003). Online control customization via optimization-based control. In Samad, T. and Balas, G., editors, Software-Enabled Control, Information technology for dynamical systems, pages 149-174. Wiley-Interscience. 
ICINCO 2008 - International Conference on Informatics in Control, Automation and Robotics

Olfati-Saber, R. (2006). Flocking for multi-agent dynamic systems: Algorithms and theory. IEEE Trans. Automat. Control, 51(3):401-420.

Simon, D. (2005). Optimal state estimation. Wiley.

Vissière, D., Chang, D. E., and Petit, N. (2007). Experiments of trajectory generation and obstacle avoidance for a UGV. In Proc. of the 2007 American Control Conference.

Xiaokui, Y. and Jianping, Y. (2002). Study on lowcost GPS/DMU integrated navigation system. In AIAA/AAS Astrodynamics Specialist Conference and Exhibit. 